\title{
Erratum: Nonlinear waves in zinc [JETP 87, 396-400 (August 1998)]
}

V. G. Skobov and A. S. Chernov

Moscow State Institute of Engineering Physics, 115409 Moscow, Russia

Zh. Éksp. Teor. Fiz. 115, 379 (January 1999)

[S1063-7761(99)03101-7]

In the article, the following sentence was omitted: "This work was supported in part by the Russian Fund for Fundamental Research (Grant No. 98-2-18393)."

Translated by M. E. Alferieff 